\title{
LATERAL COMPLETION FOR ARBITRARY LATTICE GROUPS
}

\author{
BY S. J. BERNAU ${ }^{1}$
}

Communicated by Joseph Rotman, August 2, 1973

1. Introduction. An old problem in the theory of lattice groups is to construct, for a given lattice group $G$, a canonical extension, $\hat{G}$, with the property that if $M$ is a subset of $\hat{G}$ such that $x \wedge y=0$ if $x, y \in M$ and $x \neq y$, then $M$ has a supremum in $\hat{G}$. The problem was first solved, for conditionally complete vector lattices, by Nakano [7] and was also treated by Pinsker [8] with the conditional completeness assumption.

The property required of $\hat{G}$ above is called lateral completeness. The requirement that $G$ should be canonical is met by defining the lateral completion of $G$ to be a laterally complete extension $\hat{G}$ of $G$ such that: (i) no sublattice group of $\hat{G}$ is laterally complete and contains $G$; and (ii) $G$ is dense in $\hat{G}$, which means that if $f \in \hat{G}$ and $f>0$, there exists $g \in G$ such that $0<g \leqq f$. If requirement (ii) is dropped we call $\hat{G}$ an $\mathscr{L}$-completion of $G$.

The existence and uniqueness of a lateral completion of $G$ are consequences of the orthocompletion of the author [1] in the case $G$ is representable. Conrad [5] simplified the proofs in [1] and also showed the existence and uniqueness of a lateral completion if $G$ has zero radical. Conrad's paper points out that $\mathscr{L}$-completions exist in profusion, as a consequence of the Holland representation [6], and that they can be highly pathological. Byrd and Lloyd [4] showed that Conrad's methods can be pushed through to the completely distributive case.

2. The main theorem. We have the following

THEOREM. Every lattice group $G$ has a lateral completion $\hat{G}$ which is unique up to isomorphism.

The proof of this theorem is long and technical. We outline the method.

Let $\mathscr{D}$ be the set of pairwise disjoint subsets of the lattice group $G$; i.e. $M \in \mathscr{D}$ if $\varnothing \neq M \subset G$ and $x \wedge y=0$ for $x, y \in M, x \neq y$. We first construct

AMS (MOS) subject classifications (1970). Primary 06A55; Secondary 06A50, 06A60, 06A75, 06A35.

Key words and phrases. Lateral completion, orthocompletion, radical of a lattice group, distributive radical of a lattice group, representable lattice group.

${ }^{1}$ Preparation of this paper was partially supported by NSF grant GP27916. 
a lattice group $G^{*}$ which, if we knew the existence of the lateral completion $\hat{G}$, would be the sublattice group of $\hat{G}$ generated by all $\bigvee M$ with $M \in \mathscr{D}$. This is done by considering first the semigroup $\mathscr{H}$ of $n$-tuples $\left(\varepsilon_{1} M_{1}, \cdots\right.$, $\varepsilon_{n} M_{n}$ ) for $n=1,2, \cdots$, with each $\varepsilon_{i}= \pm 1$ and each $M_{\imath} \in \mathscr{D}$, where we define

$$
\left(\varepsilon_{1} M_{1}, \cdots, \varepsilon_{n} M_{n}\right)+\left(\varepsilon_{n+1} M_{n+1}, \cdots, \varepsilon_{p} M_{p}\right)=\left(\varepsilon_{1} M_{1}, \cdots, \varepsilon_{p} M_{p}\right) .
$$

Then let $\mathscr{G}$ consist of all finite sets of finite subsets of $\mathscr{H}$. (For example, the element $\left\{\left\{\left(M_{1},-M_{2}\right)\right\},\left\{\left(M_{3}, M_{4}\right),\left(-M_{5}, M_{6}\right)\right\}\right\}$ corresponds to

$$
\left.\left(\bigvee M_{1}-\bigvee M_{2}\right) \vee\left[\left(\bigvee M_{3}+\bigvee M_{4}\right) \wedge\left(-\bigvee M_{5}+\bigvee M_{6}\right)\right] \text { in } \hat{G} .\right)
$$

Operations of addition, negative, supremum and infimum are introduced in $\mathscr{G}$. The definitions are the same as the corresponding ones in [2]. The quotient of $\mathscr{G}$ by a suitable equivalence relation turns the quotient, $G^{*}$, into a lattice group which contains a densely embedded copy of $G$ and a supremum for every (copy of) $M \in \mathscr{D}$.

We next mimic Conrad's proof for the zero radical case. Define $G(0)=G$, and for each ordinal $\beta ; G(\beta)=G(\gamma)^{* \prime}$ if $\beta=\gamma+1$, and $G(\beta)=\lim _{\gamma<\beta} G(\gamma)$ (the direct limit) if $\beta$ is a limit ordinal. Because $G$ is dense in each $G(\beta)$, we have a bound for the cardinality of pairwise disjoint subsets of the $G(\beta)$ and are able to show that $G(\beta+1)=G(\beta)$ if $\beta$ is the least ordinal whose cardinal exceeds that of $G$. It follows that $G(\beta)$ is a lateral completion of $G$.

Uniqueness is proved by taking another lateral completion of $G$ and extending the embedding of $G$ to one of $G(1)$ and so on up the chain to $G(\beta)$ by transfinite induction.

3. Structure of the lateral completion. The transfinite induction technique described above is used to prove most of the structure theorems about the lateral completion. The problem then reduces to proving the results about $G(1)$. Representability and the archimedean property are, of course, inherited by the lateral completion.

Of more interest, in view of the Conrad [5] and Byrd and Lloyd [4] results, is the relation between the radicals of $G$ and those of its lateral completion. For $g \in G$ let $R_{g}\left(L_{g}\right)$ be the solid subgroup of $G$ generated by all solid subgroups (all solid normal subgroups) of $G$ which do not contain $g$. The ideal radical $L(G)=\bigcap\left\{L_{g}: g \in G\right\}$, and the Conrad radical $R(G)=\bigcap\left\{R_{g}: g \in G\right\}$. The distributive radical $D(G)$ is the intersection of all closed prime subgroups of $G$. It is known [3] that $L(G) \subset D(G) \subset R(G)$, and that $G$ is completely distributive if and only if $D(G)=0$. An important structure theorem for the lateral completion of $G$ is the following. 
THEOREM. Let $G$ be a lattice group and $\hat{G}$ its lateral completion, then

$$
L(G) \cup(L(\hat{G}) \cap G) \subset D(\hat{G}) \cap G=D(G) \subset R(\hat{G}) \cap G=R(G) .
$$

This result shows the relationship between our construction, Byrd's and Lloyd's, and Conrad's, and how each generalizes its predecessor.

\section{REFERENCES}

1. S. J. Bernau, Orthocompletion of lattice groups, Froc. London Math. Soc. (3) 16 (1966), 107-130. MR 32 \#5554.

2. — Free non-abelian lattice groups, Math. Ann. 186 (1970), 249-262. MR 42 \#160.

3. R. D. Byrd and J. T. Lloyd, Closed subgroups and complete distributivity in latticeordered groups, Math. Z. 101 (1967), 123-130. MR 36 \#1371.

4. Soc. (2) 1 (1969), 358-362. MR 40 \#2584.

5. Paul Conrad, The lateral completion of a lattice-ordered group, Proc. London Math. Soc. (3) 19 (1969), 444-480. MR 39 \#5442.

6. Charles Holland, The lattice-ordered group of automorphisms of an ordered set, Michigan Math. J. 10 (1963), 399-408. MR 28 \#1237.

7. H. Nakano, Modern spectral theory, Maruzen, Tokyo, 1950. MR 12, 419.

8. A. G. Pinsker, Extended semiordered groups and spaces, Uchen Zapiski Leningrad Gra. Ped. Inst. 86 (1949), 236-365.

Department of Mathematics, University of TeXas, Austin, TeXas 78712 